\title{
Helioseismic Holography of an Artificial Submerged Sound Speed Perturbation and Implications for the Detection of Pre-Emergence Signatures of Active Regions
}

\author{
D. C. Braun ${ }^{1}$
}

(C) Springer $\bullet \bullet \bullet$

\begin{abstract}
We use a publicly available numerical wave-propagation simulation of Hartlep et al. (Solar Phys. 268, 321, 2011) to test the ability of helioseismic holography to detect signatures of a compact, fully submerged, $5 \%$ sound-speed perturbation placed at a depth of $50 \mathrm{Mm}$ within a solar model. We find that helioseismic holography as employed in a nominal "lateral-vantage" or "deepfocus" geometry employing quadrants of an annular pupil is capable of detecting and characterizing the perturbation. A number of tests of the methodology, including the use of a plane-parallel approximation, the definition of travel-time shifts, the use of different phase-speed filters, and changes to the pupils, are also performed. It is found that travel-time shifts made using Gabor-wavelet fitting are essentially identical to those derived from the phase of the Fourier transform of the cross-covariance functions. The errors in travel-time shifts caused by the plane-parallel approximation can be minimized to less than a second for the depths and fields of view considered here. Based on the measured strength of the mean travel-time signal of the perturbation, no substantial improvement in sensitivity is produced by varying the analysis procedure from the nominal methodology in conformance with expectations. The measured travel-time shifts are essentially unchanged by varying the profile of the phase-speed filter or omitting the filter entirely. The method remains maximally sensitive when applied with pupils that are wide quadrants, as opposed to narrower quadrants or with pupils composed of smaller arcs. We discuss the significance of these results for the recent controversy regarding suspected pre-emergence signatures of active regions.
\end{abstract}

Keywords: Helioseismology, Observations

\section{Introduction}

For almost two decades, helioseismic methods have been employed to search for evidence of magnetic flux rising through the convection zone (Braun, 1995.

${ }^{1}$ NWRA, CoRA Office, 3380 Mitchell Ln, Boulder CO 80301 USA email: dbraun@nwra.com 
Chang, Chou, and Sun, 1999;:Jensen et al., 2001; Zharkov and Thompson, 2008: Kosovichev, 2009: Hartlep et al., 2011, Ilonidis, Zhao, and Kosovichev, 2011, Leka et al., 2012: Birch et al., 2012). Submerged magnetic fields may produce travel-time anomalies due to changes in the wave speed caused by the magnetic field or by the presence of flows and perturbations to the thermal structure associated with the magnetic field (Birch, Braun, and Fan, 2010). If positively identified, such signatures could play an important role in space-weather forecasting, and lead to physical understanding of the emergence process, which is a key component of the solar activity cycle. Recent detection of $p$-mode travel-time anomalies prior to the emergence of several large active regions, obtained with time-distance methods, have been reported (Ilonidis, Zhao, and Kosovichev 2011, 2012b) No significant travel-time anomalies were subsequently measured from an independent analysis using helioseismic holography (Braun, 2012). Ilonidis, Zhao, and Kosovichev (2012a) suggest that this discrepancy may be due to differences in sensitivity between the methods employed.

Numerical simulations have provided artificial data through which helioseismic analysis and modeling can be tested (Jensen et al., 2003, Benson, Stein, and Nordlund, 2006: Hanasoge et al., 2006, Parchevsky and Kosovichev, 2007; Zhao et al., 2007; Braun et al., 2007: Cameron, Gizon, and Duvall, 2008, Parchevsky and Kosovichev, 2009, Crouch et al., 2010. Cameron et al., 2011; Birch et al., 2011; Hartlep et al., 2011; Braun et al., 2012). Many of these simulations include near-surface flows, sound-speed perturbations, or magnetic structures typical of active regions or supergranulation. Simulations that propagate waves through completely submerged perturbations are rarer (Hartlep et al., 2011), but are critical for testing and developing helioseismic methods sensitive to looking for active regions prior to their emergence on the surface. In this work, we use one of the simulations of Hartlep et al. (2011) to test the sensitivity of helioseismic holography comparatively to subsurface sound-speed perturbations under a variety of applications.

\section{Simulation}

Hartlep et al. (2011) constructed a number of simulations containing $p$ modes propagating through a spherical domain containing localized perturbations of the sound speed about the standard solar Model S (Christensen-Dalsgaard et al., 1996). No flows or magnetic fields are included. The solar model is convectively stabilized by a neglect of the entropy gradient of the background model, which lowers the acoustic cut-off frequency. The mode amplitudes above $3.5 \mathrm{mHz}$ are thus reduced in amplitude. In addition, the simulation is only populated with $p$ modes with angular degree $\ell$ between 0 and 170 . The simulations span about 17 hours of solar time. A number of simulations using the same code are publicly available and include a variety of sound-speed perturbations at different depths. In this work, we employ the simulation with a peak $5 \%$ soundspeed reduction at a depth of $50 \mathrm{Mm}$ and with a horizontal size of $45 \mathrm{Mm}$ (see Figure 1). The simulated velocity field is provided in arbitrary units and represented in heliographic coordinates, with 512 pixels in longitude and 256 

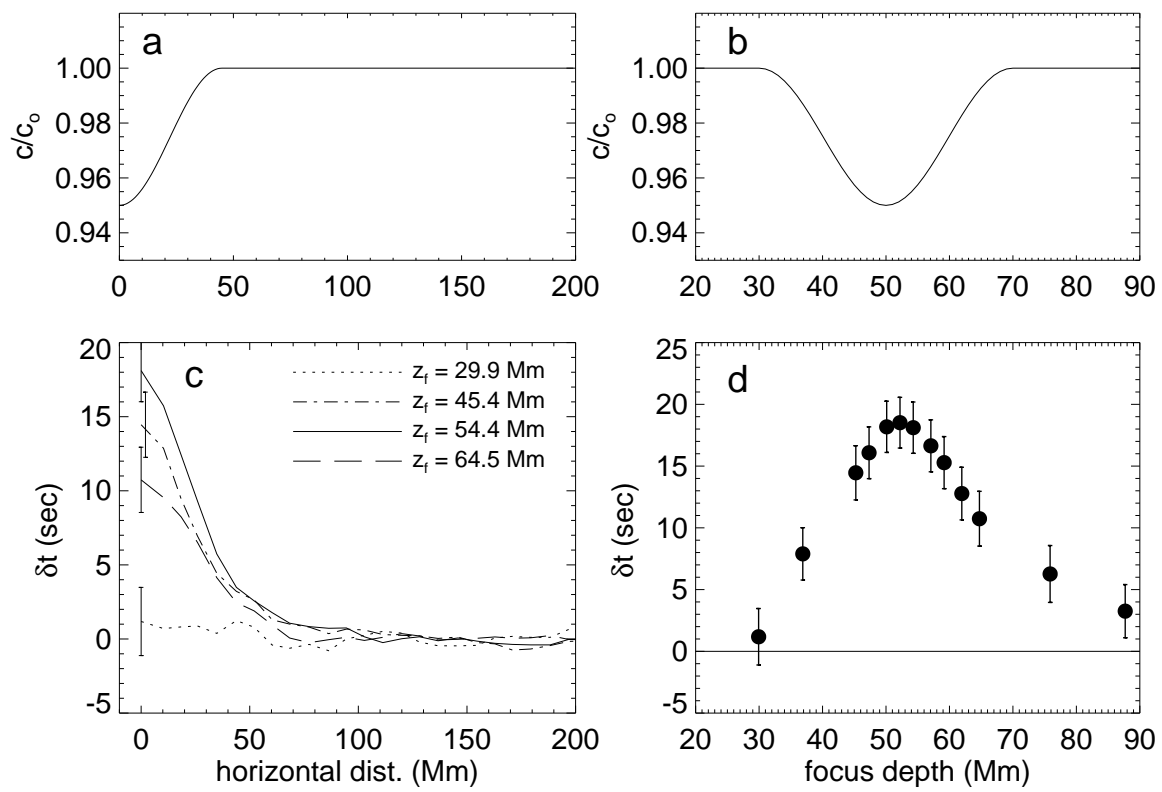

Figure 1. The sound-speed ratio $\left[c / c_{o}\right]$ in the simulation, where $c$ is the perturbed sound speed and $c_{O}$ is the background sound speed of Model S (top panels), and travel-time measurements (bottom panels) made with helioseismic holography using a "nominal" methodology (see text). (a) The variation with horizontal distance from the center of the circularly symmetric sound-speed perturbation at a depth of $50 \mathrm{Mm}$ below the surface of the simulation. (b) The variation with depth of the sound-speed ratio at the center of the perturbation. (c) The variation with horizontal distance of the azimuthally averaged mean travel-time shift measured using lateral-vantage helioseismic holography applied to the simulation at focus depths of 29.9 Mm (dotted line), 45.4 Mm (dash-dot line), 54.4 Mm (solid lines), and 64.5 Mm (long dashed line). The travel-time shifts are averaged over $0.7^{\circ}$-wide annuli centered on the location of the perturbation. (d) The travel-time shift at the center of the perturbation as a function of focus depth. The error bars in panels (c) and (d) indicate the standard deviation of the realization noise determined from a region away from the perturbation (see text).

pixels in latitude, a cadence of one minute, and it is stored in a FITS file (see sun.stanford.edu/ thartlep/Site/Artificial_Data/Entries/2012/3/21_Subsurface_sound_speed_perturbations.html

Our primary emphasis is on testing the ability of helioseismic holography to detect $p$-mode travel time signatures of the prescribed perturbation within the simulation, and to measure the relative sensitivity of the results (in both signal strength and background noise) to changes of methodology. In contrast, direct comparisons of measured and expected travel-times requires the computation and application of sensitivity functions which is not attempted here. A prediction of the travel-time shift expected from a given sound-speed perturbation is a nontrivial exercise, but a rough estimate is useful. We estimate the travel-time shift in the geometric optics limit as the path integral of the fractional sound-speed perturbation (Equation 1 of Hartlep et al. . 2011) weighted by the inverse of the background sound speed in Model S. For convenience, the path is chosen as purely horizontal through the center of the perturbation. This procedure yields a travel-time increase of 23 seconds. 


\section{The Nominal Procedure and Results}

Helioseismic holography (hereafter HH) is described extensively elsewhere (Lindsey and Braun. 1997, Chang et al., 1997, Lindsey and Braun, 2000 2004). For our purposes, it is useful to enumerate the data-analysis steps taken to define the "nominal," or baseline, procedure. This provides the context for investigating the sensitivity of the results to changes in methodology discussed in Section 4

The basic idea is to apply Green's functions to the solar oscillation field at the surface of the Sun (or in this case, a simulation) to estimate the amplitudes of incoming and outgoing waves at targets (or "focal points") at or below the surface. In the "lateral-vantage" or "deep-focus" configuration of HH (Lindsey and Braun, 2004, Braun, Birch, and Lindsey, 2004:Braun and Birch, 2008a), travel-time perturbations are extracted from the cross-covariances between these "ingression" and "egression" amplitudes with a focus below the surface (Figure 2].

To establish some common notation, we define the three-dimensional (3D) Fourier transform in time $[t]$ and two spatial dimensions $[x, y]$ of a function $A(x, y, t)$ as $\hat{A}\left(k_{x}, k_{y}, \omega\right)$ where $k_{x}$ and $k_{y}$ are the horizontal wavenumber components and $\omega$ is the temporal frequency. We define the Fourier transform in only the temporal dimension of $A$ as $\tilde{A}(x, y, \omega)$. With this, the steps involved in the data analysis are listed below.

i) The simulated surface-velocity data, provided in heliographic coordinates, are remapped onto a Postel projection $\Psi(x, y, t)$. The nominal spacing of the Postel grid is $\delta x=\delta y=8.54 \mathrm{Mm}\left(0.7^{\circ}\right)$, which is the original spacing of the velocity data in heliographic coordinates. The central tangent point $(x, y)=(0,0)$ is defined as $34.2 \mathrm{Mm}\left(2.8^{\circ}\right)$ south of the location of the perturbation.

ii) The 3D Fourier transform $\left[\hat{\Psi}\left(k_{x}, k_{y}, \omega\right)\right]$ of the Postel-projected data is computed in both spatial dimensions and in time. In the temporal-frequency domain, the data within a bandpass of 2.5 and $5.5 \mathrm{mHz}$ are extracted for further analysis. We note that the simulation contains very little $p$-mode power above $3.5 \mathrm{mHz}$.

iii) A phase-speed filter is applied to $\hat{\Psi}$. The nominal method employs filters that are Gaussian in the phase speed $\left[w \equiv \omega / k\left(\right.\right.$ where $\left.\left.k^{2}=k_{x}{ }^{2}+k_{y}{ }^{2}\right)\right]$ for each depth with peak phase speeds $\left[w_{o}\right]$ and widths $[\delta w]$ specified in Table 1 . In Section 4.3 we examine the sensitivity of the results to variations in the form of the filter.

iv) A set of depths is chosen (Table 1) and Green's functions for both diverging $\left[G_{+}^{P}\right]$ and converging $\left[G_{-}^{P}\right]$ waves are computed in the same Postel projected grid as the data (Lindsey and Braun, 2000). The Green's functions are multiplied by spatial masks defining a given pupil $[P]$. The nominal set of pupils represent quadrants (or "arcs") of annuli extending outward in four directions and are denoted $\mathrm{E}, \mathrm{W}, \mathrm{N}$, and $\mathrm{S}$. The annulus widths are determined by ray theory from the paths of acoustic modes diverging from 


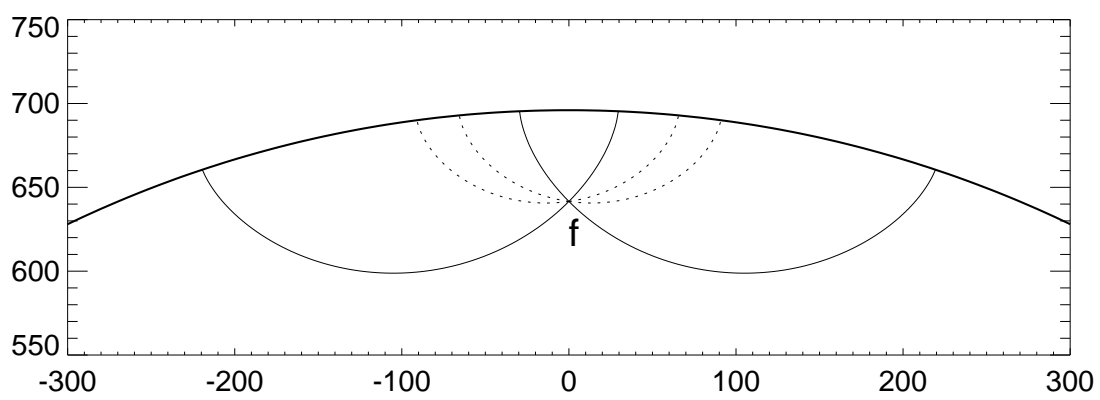

Figure 2. Ray paths for $p$-modes converging on a focal point $f$ that is $54.4 \mathrm{Mm}$ below the surface of the spherical domain of the simulation (denoted by the thick solid line). The thinner solid lines denote rays spanning $\pm 45^{\circ}$ from the horizontal direction. This is the nominal range of impact angles for lateral-vantage helioseismic holography. In Section 4.4 we perform $\mathrm{HH}$ with smaller ranges of impact angles. The dashed lines indicate the ray paths for impact angles spanning $\pm 7.5^{\circ}$ from the horizontal direction. The scale is in $\mathrm{Mm}$.

the subsurface focus point and spanning a range of "impact angles" $\pm 45^{\circ}$ from the horizontal direction (see Figure 2). In Section 4.4 we explore the sensitivity of the results to narrower ranges of impact angles and in Section 4.5 we employ different azimuthal extents of the pupil arcs.

v) For each pupil quadrant $[P]$, the ingression $\left[H_{-}^{P}\right]$ and egression $\left[H_{+}^{P}\right]$ amplitudes are estimated by convolutions of the data cube $[\Psi]$ with $G_{-}^{P}$ and $G_{+}^{P}$, respectively, in both time and the two spatial coordinates. This is performed using a plane-parallel approximation by the simple product of $\hat{G}_{ \pm}^{P}$ and $\hat{\Psi}$ (Lindsey and Braun, 2000) in the three-dimensional Fourier domain. The validity and consequences of this approximation are explored in Section 4.1 .

vi) The cross-covariance functions between ingression and egression amplitudes corresponding to opposite quadrants (e.g. E and W, N and S) are computed. The four resulting cross-covariance functions are summed.

vii) Mean travel-time maps are determined from the sum of the four crosscovariance functions. The nominal method uses the "phase method" (Braun and Lindsey, 2000). In Section 4.2 we compare the phase method with results from fits of the cross-covariances to Gabor wavelets.

viii) Maps of the mean travel-time shifts are determined from the residual of the travel-time maps after subtracting a two-dimensional polynomial fit to a "quiet-Sun" area excluding the perturbation. As shown in Section 4.1 this procedure helps to remove the effects of the plane-parallel approximation used in step $\mathbf{\nabla})$.

Table 1 shows the pupil ranges for each selected focus depth $z_{f}$, determined from ray theory. Also listed in the table are the range of mode degrees $[\ell]$ at 3 $\mathrm{mHz}$, sampled by the pupil, and the parameters for the Gaussian phase-speed 
Table 1. Pupil sizes, modes, and filter parameters

\begin{tabular}{ccccc}
\hline $\begin{array}{c}z_{f} \\
{[\mathrm{Mm}]}\end{array}$ & $\begin{array}{c}\text { Pupil Radii } \\
{[\mathrm{Mm}]}\end{array}$ & $\ell$ at $3 \mathrm{mHz}$ & $\begin{array}{c}w_{0} \\
{\left[\mathrm{~km} \mathrm{~s}^{-1}\right]}\end{array}$ & $\begin{array}{c}\delta w \\
{\left[\mathrm{~km} \mathrm{~s}^{-1}\right]}\end{array}$ \\
\hline 29.9 & $16.0-128$ & $124-175$ & 74 & 37 \\
37.0 & $19.5-159$ & $108-153$ & 87 & 43 \\
45.4 & $24.4-190$ & $95-134$ & 96 & 49 \\
47.6 & $25.8-195$ & $92-130$ & 101 & 50 \\
49.9 & $27.1-209$ & $89-126$ & 105 & 52 \\
52.4 & $28.5-216$ & $86-122$ & 108 & 54 \\
54.4 & $29.2-224$ & $84-119$ & 111 & 55 \\
57.1 & $31.3-230$ & $82-115$ & 114 & 57 \\
59.2 & $32.0-237$ & $79-113$ & 117 & 58 \\
62.1 & $34.1-251$ & $77-109$ & 119 & 60 \\
64.5 & $36.2-254$ & $75-106$ & 122 & 62 \\
76.1 & $41.8-292$ & $67-95$ & 140 & 69 \\
87.9 & $48.0-327$ & $60-85$ & 153 & 77 \\
\hline
\end{tabular}

filter (see Section 4.3) at each depth. The highest value of $\ell$ at each depth represents waves propagating horizontally through the focal point while the lowest value indicates modes which propagate at impact angles of $\pm 45^{\circ}$ from the horizontal direction (see Figure 2).

Figure 3 shows maps of the travel-time shifts for a sample of focus depths. The perturbation is clearly seen as an increase in travel-time shift with a maximum of between 15 and 20 seconds at the expected horizontal position. Figure 1 ; shows the azimuthal averages of the travel-time shifts for several focus depths while Figure $1 \mathrm{~d}$ shows the variation of the travel-time shift at the center of the perturbation (hereafter "peak travel-time shift") with focus depth. It is clear that the horizontal and vertical dependence of the travel-time shifts reasonably characterizes the shape of the perturbation.

We measure a background realization noise $[\sigma]$ as the standard deviation of the mean travel-time shifts within an annulus spanning distances 111-195 Mm from the center of the Postel projection. For the "nominal" maps shown in Figure 3 , $\sigma$ is about 2.1 seconds and does not vary substantially with depth. We find that the background noise for maps made at different focus depths is correlated. For example, there is a correlation coefficient (measured after excluding a region around the perturbation) of 0.96 between maps made at 54.4 and $52.4 \mathrm{Mm}$, and a correlation of 0.56 between maps at 54.4 and $47.6 \mathrm{Mm}$.

\section{Tests of the Methodology}

\subsection{Tests of the Plane-parallel Approximation}

In Section 3 step $\mathbf{v})$, a convolution in time and horizontal spatial coordinates between the Green's functions $G_{ \pm}^{P}$ and the data $\Psi$ is computed in the Fourier 
a
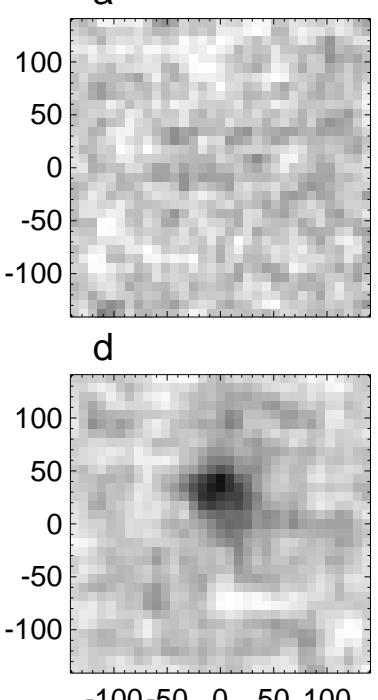

b

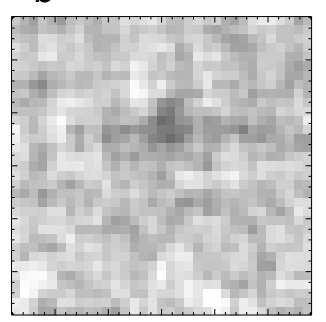

e

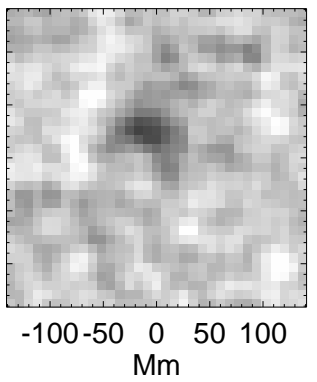

C

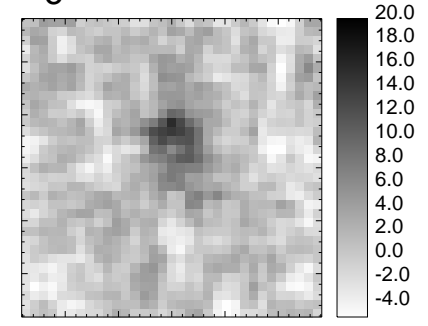

f

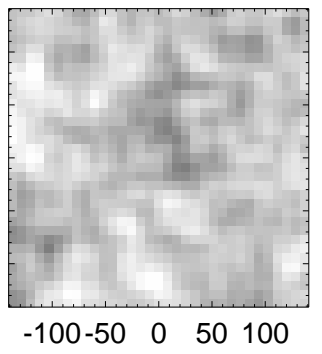

Figure 3. Maps of the mean travel-time shift using the nominal methodology of lateral-vantage $\mathrm{HH}$ as applied to the Hartlep et al. simulation and for focus depths of: (a) $29.9 \mathrm{Mm}$, (b) 37.0 Mm, (c) 45.4 Mm, (d) 54.4 Mm, and (e) 64.4 Mm. The greyscale indicates the travel-time shift in seconds.

domain under the assumption that the functions $G_{ \pm}^{P}$ are invariant with respect to translation in the Postel coordinate frame (this assumption has been termed the "plane-parallel" approximation; Lindsey and Braun, 2000, Braun and Birch, 2008b). The use of this approximation is highly desirable since it decreases computing time and resources by several orders of magnitude. Without its use, for example, separate Green's functions for each target pixel would have to be computed, stored, accessed, and operated on with a 3D multiplication by the datacube in the computations.

A major result of this approximation is the introduction of a systematic bias in the mean travel-time shift, which is a function of the horizontal distance between the focus and the central tangent point of the Postel projection. The reason for this bias is straightforward to understand: In the Postel (also known as azimuthal equidistant) projection, distances measured along any line intersecting the central tangent point (hereafter simply called the "center") are accurate, but distances between all other points differ from their true great-circle values. Thus, a locus of constant phase of waves propagating either away from or towards the center is warped in the projected plane into an ellipse with the semi-minor axis aligned towards the center (Figure 4). These wavefronts do not match the assumed circular wavefronts (and pupils) of the computed Green's functions. For the depths and pupil parameters listed in Table 1 the distortion in distance is small compared to the horizontal wavelengths of the modes. For example, in Figure 4 are drawn wavefronts at the outer pupil boundaries (where the 

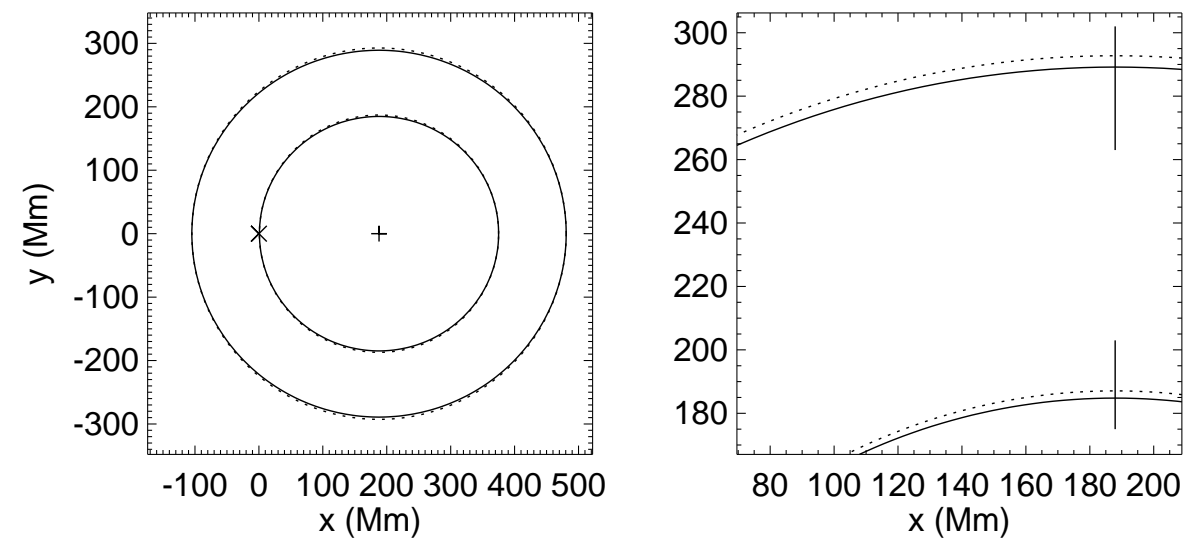

Figure 4. Examples of the distortion in wavefronts at the outer pupil boundaries centered on a target focus point (denoted by the plus sign) placed $188 \mathrm{Mm}$ to the right of the center of the Postel projection which is denoted by the $\times$ symbol. The larger and smaller dotted circles show wavefronts at the outer pupil boundaries, as projected onto the Postel frame, for focus depths of 76.1 and $45.4 \mathrm{Mm}$ respectively. The solid circles show circular wavefronts as assumed in the plane-parallel approximation. The dotted and solid wavefronts coincide along the $x$-axis but deviate at other places, with the maximum deviation occurring at the the top and bottom. The deviations are difficult to discern by eye in the left panel. The right panel shows a magnified version of the upper part of the left panel. The vertical line segments in the right panel indicate the length of the horizontal wavelengths of modes that propagate from the focus depth to the outer pupil boundaries.

distortion is greatest) corresponding to focus positions placed $188 \mathrm{Mm}\left(15.5^{\circ}\right)$ to the right of the center and at depths of 45.4 and $76.1 \mathrm{Mm}$. The maximum distortion of the wavefronts for these depths is 2.3 and $3.6 \mathrm{Mm}$ respectively and this is small compared to the horizontal wavelengths $(30$ and $40 \mathrm{Mm})$ of the modes considered. However, the distortion in projected distances results in observable spurious mean travel-time variations that vary with the azimuthal angle of propagation from the focus as well as the distance between the focus and the wavefront. At the outer edge of the pupils these spurious shifts can be as large as 20-30 seconds. However, the net travel-time shift as assessed over the entire pupil is typically below ten seconds over tangent-point distances below $200 \mathrm{Mm}$ (e.g. Figure 5).

To correct for this spatially varying bias, we fit and subtract a two-dimensional polynomial to the raw mean travel-time maps (Section 3 step viii). A circular mask excluding the perturbation is applied before the polynomial fit. Figure 5 shows cuts through a mean travel-time map with and without this correction.

Since all of the distortions introduced by the plane-parallel approximation increase with tangent-point distance, it is worthwhile to test the approximation by computing travel-time shift maps with varying positions of the tangent point. A similar test was performed by Braun (2012) on solar observations, but comparing only measurements of realization noise. The present simulation provides a larger, isolated, signal that provides a complementary target for this type of test. Figure 6 shows that maps made using tangent points spaced $200 \mathrm{Mm}$ apart show, after correction for the bias discussed above, have residual differences on 


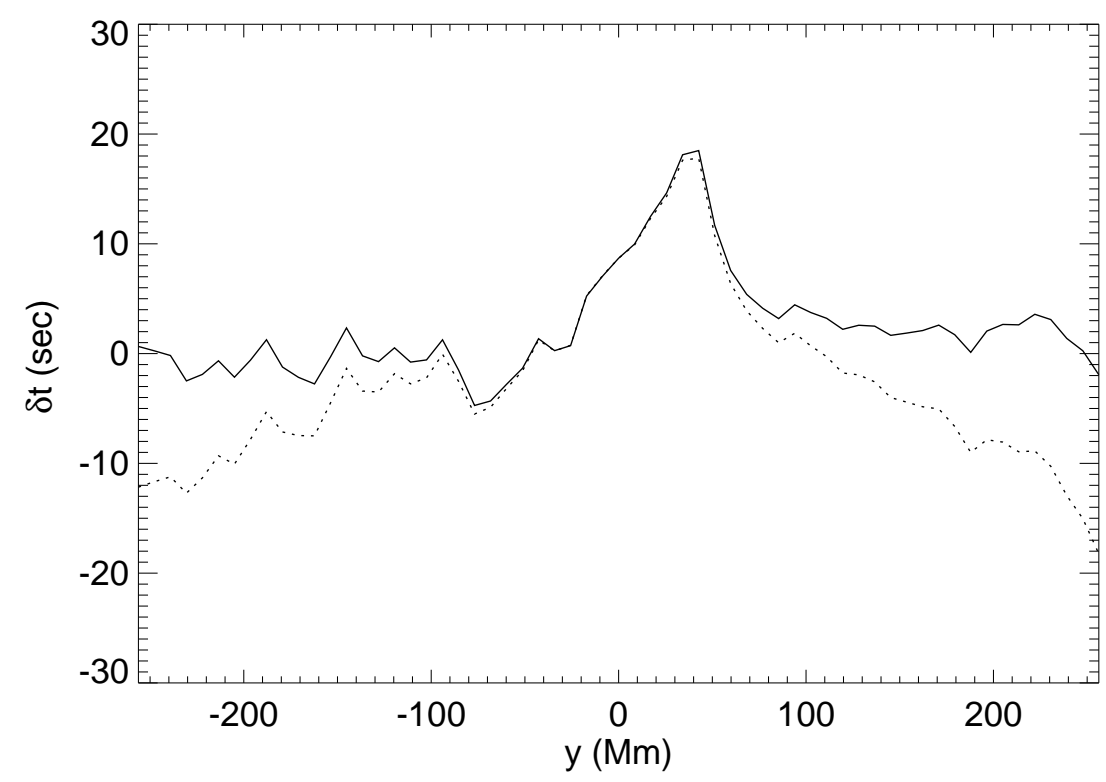

Figure 5. Comparison of mean travel-time shifts with and without a polynomial subtraction designed to remove the effects of the plane-parallel approximation. The dotted (solid) line is a vertical cut (at $x=0$ ) of the uncorrected (corrected) mean travel-time map at a focus depth of $54.4 \mathrm{Mm}$.

the order of a second. For smaller distances of approximately 20-30 Mm these residuals are well below a tenth of a second.

\subsection{Comparisons of Travel-time Measurements}

As we note in Section 3 step viil the mean travel times are determined from the sum of the cross-covariance functions. In the nominal procedure, there are four cross-covariances of the form

$$
\tilde{C}^{E W}\left(\mathbf{r}, z_{f}, \omega\right)=\tilde{H}_{+}^{E}\left(\mathbf{r}, z_{f}, \omega\right) \tilde{H}_{-}^{W *}\left(\mathbf{r}, z_{f}, \omega\right),
$$

where the asterisk denotes the complex conjugate, $\mathbf{r}=(x, y)$, and we have also included the dependence on focus depth $z_{f}$. The temporal Fourier transform of the sum,

$$
\tilde{C}=\tilde{C}^{E W}+\tilde{C}^{N S}+\tilde{C}^{W E}+\tilde{C}^{S N},
$$

is used in the "phase method" (Braun and Lindsey, 2000, Braun and Birch, 2008b) to compute the mean travel time through

$$
\tau_{\mathrm{pm}}\left(\mathbf{r}, z_{f}\right)=\arg \left(\left\langle\tilde{C}\left(\mathbf{r}, z_{f}, \omega\right)\right\rangle_{\Delta \omega}\right) / \omega_{o},
$$

where the brackets indicate the average over the bandwidth $\Delta \omega$, and $\omega_{0}$ is the mean frequency. The desired travel time shift $[\delta t]$ is obtained from $\tau_{\mathrm{pm}}$ 

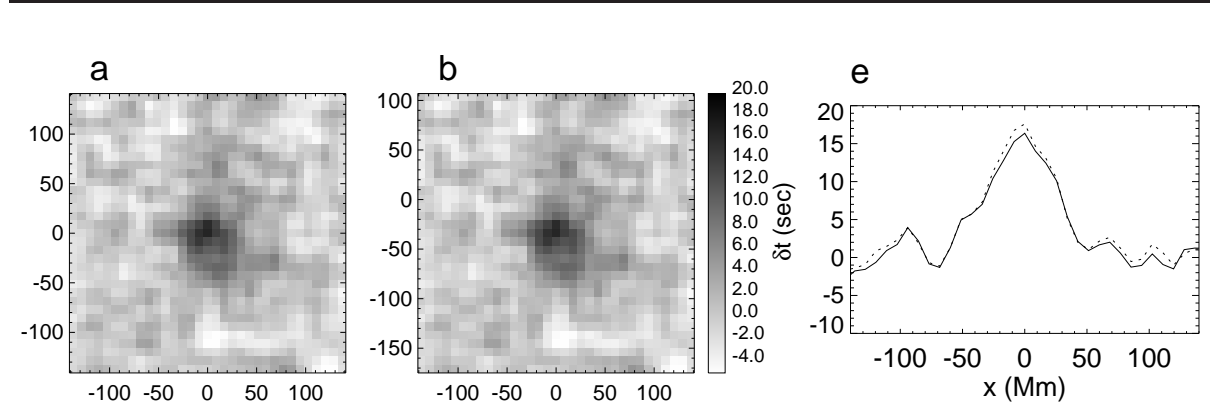

C
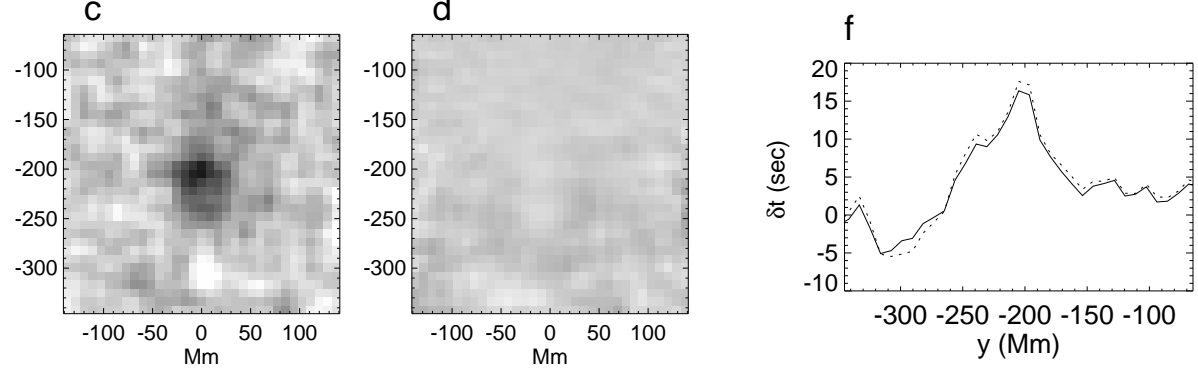

Figure 6. Maps of the mean travel-time shifts at a focus depth of $54.4 \mathrm{Mm}$ and with the tangent point (center) of the Postel frame placed at the following locations: (a) centered on the perturbation, (b) $34 \mathrm{Mm}$ to the North of the perturbation, and (c) $205 \mathrm{Mm}$ to the North of the perturbation. (d) The difference between the maps shown in (c) and (a). The rightmost plots show horizontal (e) and vertical (f) cuts through the center of the perturbation of map (a) shown as solid lines and map (c) shown as dotted lines.

by subtracting a $2 \mathrm{D}$ polynomial fit to a quiet-Sun region (step viii). A typical summed cross-covariance function, transformed back to the temporal domain, is shown in Figure 7.

An alternative method for extracting travel-time shifts is to fit the crosscovariance function to a Gabor wavelet:

$$
g=A \cos \left(\omega_{0}\left[t-\tau_{\mathrm{gf}}\right]\right) \exp \left(-\frac{1}{2}\left[\frac{t-\tau_{\mathrm{en}}}{\sigma}\right]^{2}\right)
$$

where $A, \sigma$, and $\tau_{\text {en }}$ are the amplitude, width, and position of a Gaussian envelope, $\omega_{0}$ is the mean frequency, and $\tau_{\mathrm{gf}}$ is the (phase) travel time which is used instead of $\tau_{\mathrm{pm}}$ to determine the travel-time shift $\delta t$. We have applied MPFIT routines (Markwardt, 2009) to perform a non-linear least squares fitting of the summed cross-covariance functions to Gabor wavelets for a focus depth of 54.4 $\mathrm{Mm}$. The initial guesses of $\tau_{\mathrm{gf}}$ in the fits were based on the peak closest to $t=0$ of the cross-covariance function. Figure 7 shows an example of the fit of a single cross-covariance. Figure 8 shows that there is remarkable agreement between the mean travel time shifts as determined from the phase method and the Gabor fits. We note that fine tuning the initial guesses based on the peaks of the cross-covariance functions to the left (or right) of the central peak yields phase times $\tau_{\text {gf }}$ which agree to within a fraction of a second of the times obtained using the central peak minus (or plus) the period $2 \pi / \omega_{0}$. Thus, due to statistical 


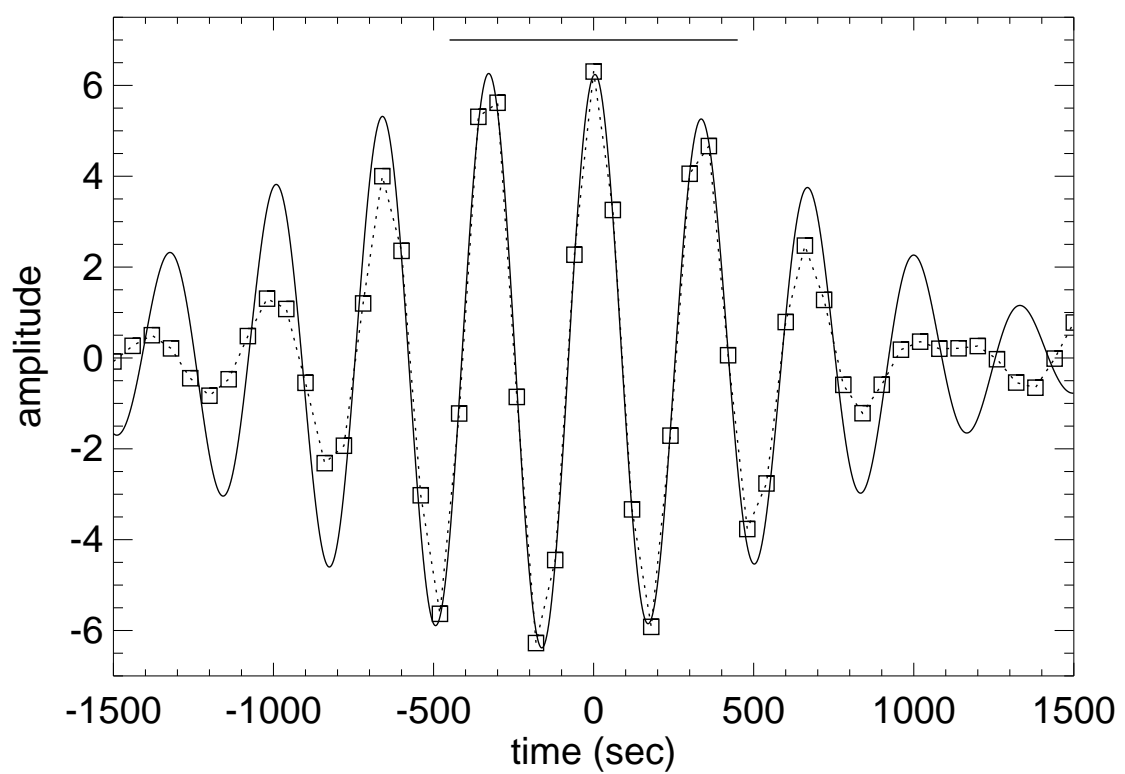

Figure 7. The squares connected by dotted lines show a cross-covariance function between the ingression and egression amplitudes, summed over the four opposite-quadrant pairs, for a single spatial location and a focus depth of $54.4 \mathrm{Mm}$. The solid curve represents a fit to the cross-covariance function, sampled over a 14-minute window denoted by the horizontal line at the top, to a Gabor wavelet (Equation 4). The amplitude is in arbitrary units.

fluctuations in the mean frequency, maps made using fits to these peaks are noisier than maps made using the central peak.

\subsection{Sensitivity to Different Phase-speed Filters}

In Section 3 step [iii), a phase-speed filter is applied to the Fourier transform $\hat{\Psi}$ of the datacube. Phase-speed filters are widely used in both time-distance helioseismology (Duvall et al., 1997; Couvidat and Birch, 2006) and helioseismic holography (Braun and Birch, 2006). The nominal procedure for lateral-vantage HH uses Gaussian filters

$$
\phi=\exp \left(-\frac{1}{2}\left[\frac{w-w_{o}}{\delta w}\right]^{2}\right)
$$

with a peak phase speed $\left[w_{o}\right]$ and width $[\delta w]$ such that square of the filter has values of one and one-half at the highest and lowest wavenumbers respectively at $3 \mathrm{mHz}$ as listed in Table 1 . It is recognized that the use of phasespeed filters reduces the noise contributed by convective (non-wave) motions as well as from $p$-modes outside the range of desired phase speeds. Recently, Ilonidis, Zhao, and Kosovichev (2012a, 2012b) claim that different types of filters can affect the measured strength of subsurface signatures of emerging active regions. We have compared results using the nominal Gaussian filter, results using 

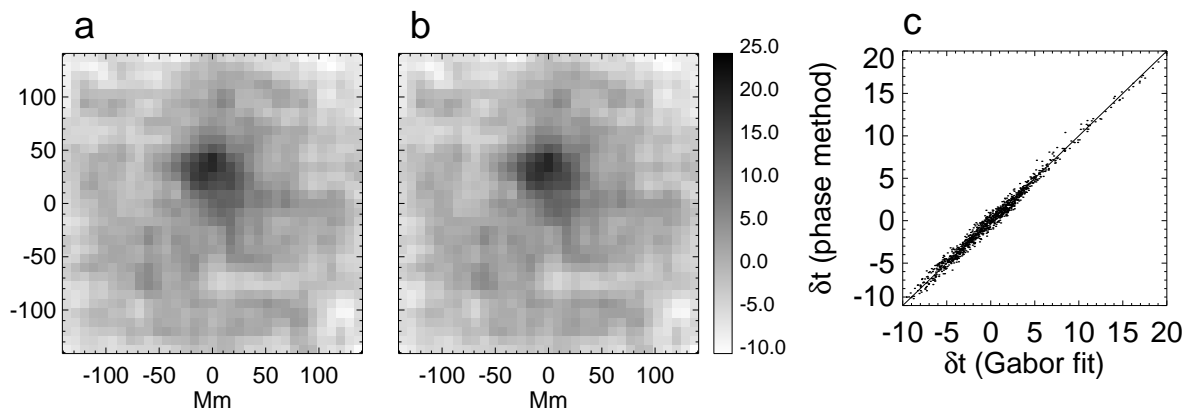

Figure 8. Comparisons of maps of mean travel-time shifts for a focus depth of $54.4 \mathrm{Mm}$ obtained (a) using the nominal method including the phase-method for extracting travel times from the cross covariance functions and (b) using fits to Gabor wavelets to the same cross-covariance functions in the temporal domain. No corrections for the bias introduced by the plane-parallel approximation have been performed here. Rather, a simple mean has been subtracted from each map. (c) A scatter plot of the two maps, compared to a line with unit slope.

a "flat-top" filter similar to that employed by Ilonidis, Zhao, and Kosovichev (2012a), and results using no phase-speed filter. Figures 9 and 10 show comparable peak travel-time shifts in the simulated perturbation between the three cases, although the flat-top filter may be somewhat less sensitive to the variation with depth of the perturbation. This is also confirmed by computing the correlation coefficient between maps for different depths. For example, traveltime shifts at focus depths of 54.4 and 64.5 have a correlation of 0.45 using the Gaussian filter, but 0.64 using the flat-top filter. These correlation coefficients were computed with the perturbation masked out, so they measure correlations in the background realization noise. Consistently higher correlation coefficients for all of the flat-top filtered results over this depth range $(45-65 \mathrm{Mm})$ are observed. In general, the use of either filter produces somewhat less noise (as determined from the standard deviation of the realization noise outside of the perturbation) than using no filter, as expected (Figure 10d).

A restriction in the simulation to mode power below $\ell=170$ means that the tests performed here are not sensitive to variations in the filter properties below $w=70 \mathrm{~km} \mathrm{~s}^{-1}$. Nonetheless, our general findings are consistent with expectations based on experience analyzing solar data for lateral-vantage holography performed for similar focus depths.

\subsection{Sensitivity to Different Quadrant Widths}

We explored the effect of changes to the range of impact angles of $p$ modes interacting with the perturbation, by decreasing the pupil width from the nominal values in Table 1, New pupil widths were computed using ray theory for impact angle extrema of $\pm 25^{\circ}, \pm 15^{\circ}$, and $\pm 7.5^{\circ}$ at the focus depth of $54.4 \mathrm{Mm}$. Figure 2 shows rays corresponding to impact angles of the nominal $\pm 45^{\circ}$ and the smallest range, $\pm 7.5^{\circ}$, considered. 


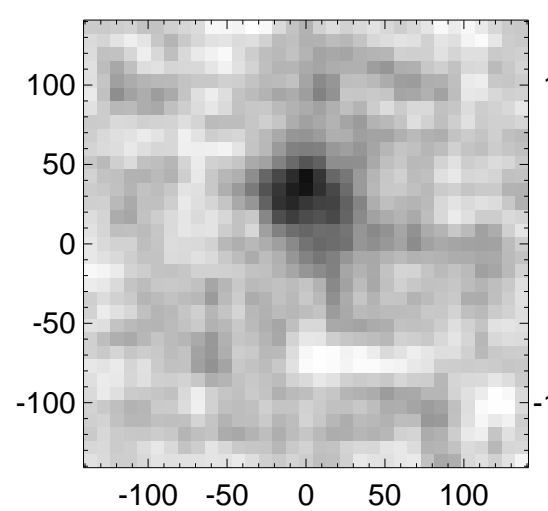

C

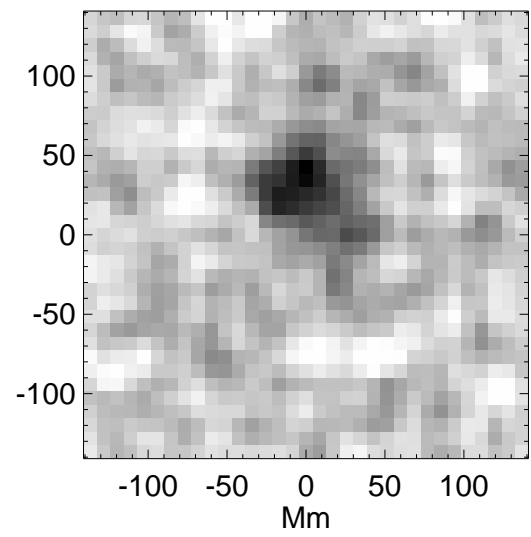

b

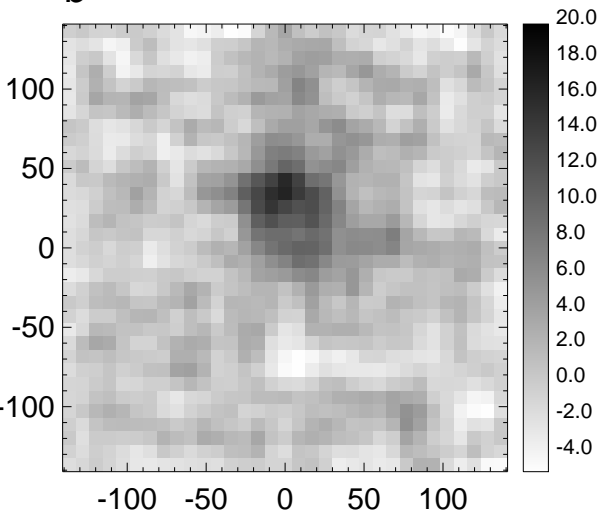

d

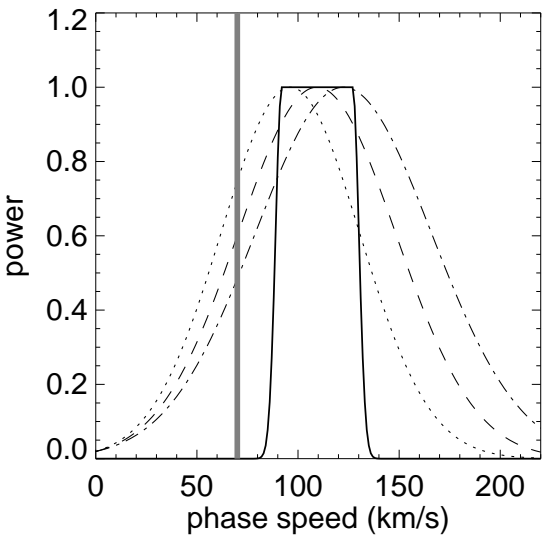

Figure 9. Mean travel-time shift maps made for a focus depth of $54.4 \mathrm{Mm}$ and different phase-speed filtering: (a) a Gaussian phase-speed filter, (b) a flat-top filter, and (c) no phase-speed filtering. (d) The square of the filter function for several filters used: dotted, dashed and dot-dash lines indicate the nominal Gaussian filters corresponding to focus depths of 45., 54.4, and 64.5 Mm respectively. The solid line shows the flat-top filter used in this study. There is no $p$-mode power in the simulation to the left of the vertical grey line. Thus, the tests here are not sensitive to differences between the filters at these low phase speeds.

Figure 11 shows that there is no substantial change in the strength of the perturbation as the impact angle is changed, within the uncertainty specified by the background realization noise. This result is expected since the travel-time shifts due to a simple sound-speed perturbation should not depend on impact angle. There is a slight increase of realization noise, which also appears to take on a more fine-scale oscillatory pattern, as the pupil quadrant widths are decreased (Figure 110). This is likely a diffraction (side-lobe) artifact due to the narrow pupil. The width of the pupils for these angles $\left( \pm 7.5^{\circ}\right)$ are smaller than the horizontal wavelength of the modes. To resolve this fine structure, the travel- 

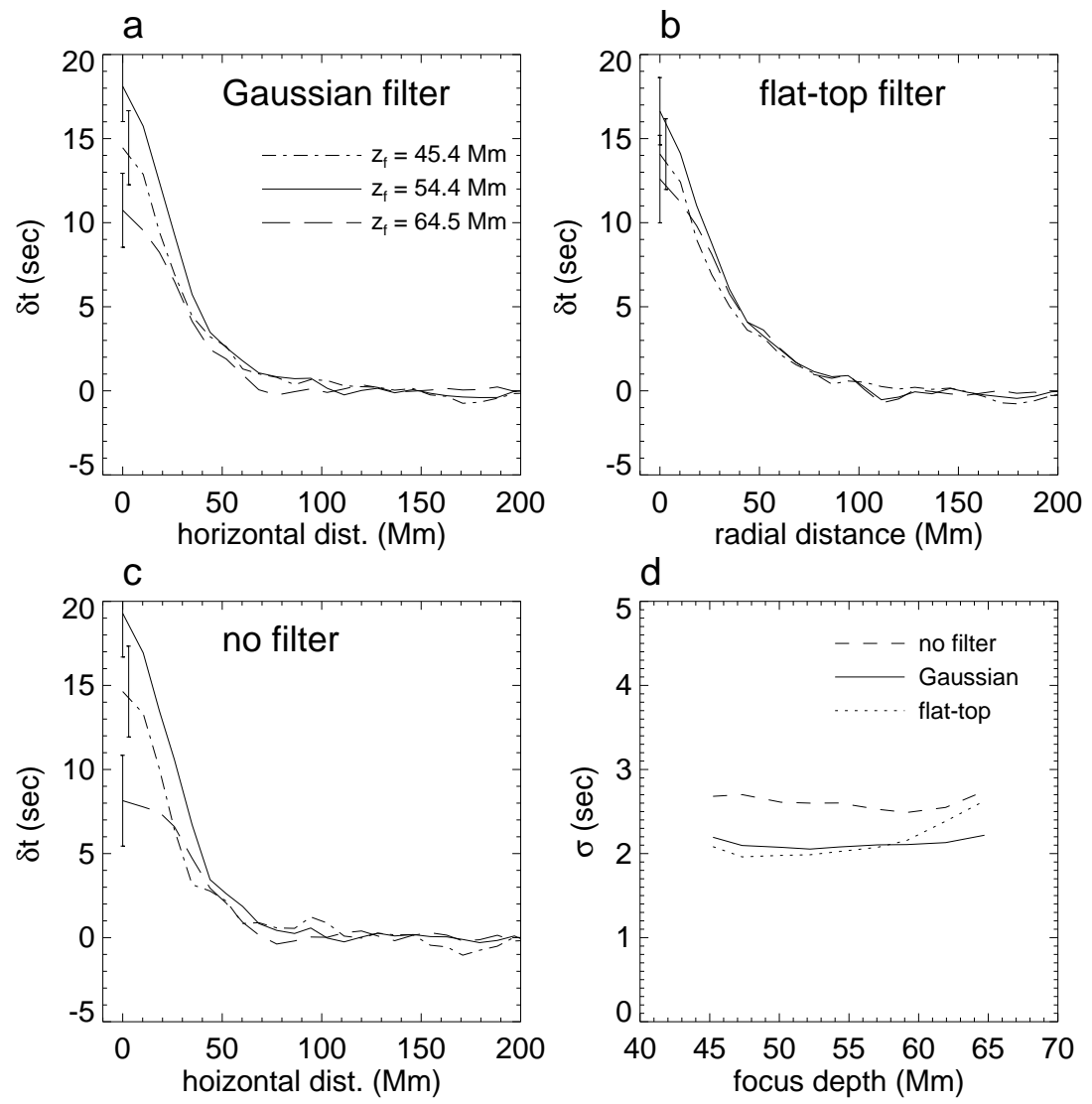

Figure 10. Mean travel-time shifts for different focus depths, averaged in annuli centered on the perturbation (as in Figure 1 k, using three different types of phase-speed filtering: (a) the nominal Gaussian filters, (b) the flat-top filter, and (c) no filtering. The depths are 45.3 (dash-dot line), 54.4 (solid lines) and 64.5 Mm (long dashed line). Error bars represent the standard deviation $[\sigma]$ of the background realization noise in a region surrounding the perturbation (see text). The variation of $\sigma$ with focus depth is shown in panel (d) for the three cases: Gaussian (solid line), flat-top (dotted line), and no filter (dashed line).

time shift maps shown in Figure 11 were made with a grid spacing of half of the nominal value, resulting from an application of a Fourier interpolation of the original data.

\subsection{Sensitivity to Pupil Arc Size}

The advantage of using four quadrants to compute the ingression/egression crosscovariances derives primarily from the utility in making measurements sensitive to flows as well as perturbations producing mean (horizontal-direction-averaged) 

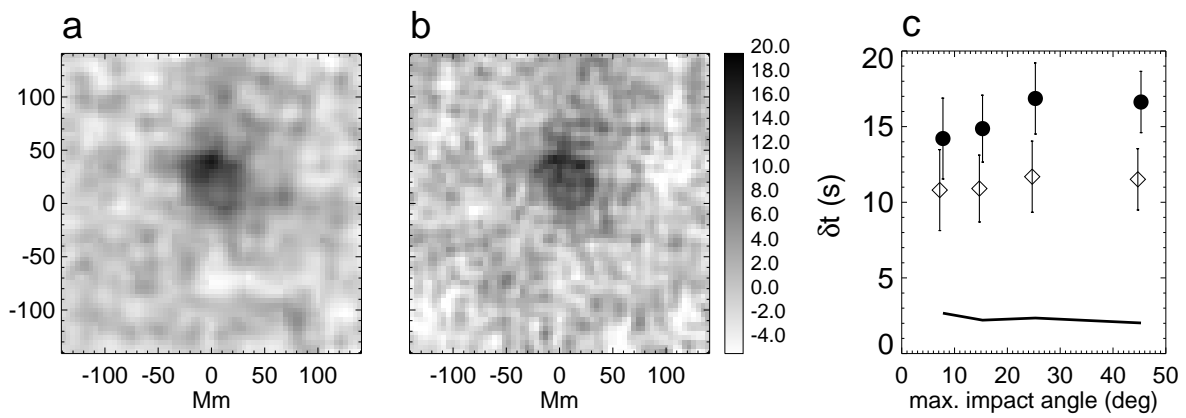

Figure 11. Mean travel-time shift maps made for a focus depth of $54.4 \mathrm{Mm}$ and with different ranges of impact angle: (a) $\pm 45^{\circ}$ and (b) $\pm 7.5^{\circ}$. Both maps were made using the same flat-top filter shown in Figure 9 (c) Measurements of the mean travel-time shift in the perturbation against the maximum (absolute) impact angle. The filled circles show the peak shift and the diamonds show the average shift within a $25 \mathrm{Mm}$ radius. Error bars denote the standard deviation of the realization noise, which is also plotted as a solid line.

travel-time shifts (Gizon and Birch, 2005). Ilonidis, Zhao, and Kosovichev (2012a, 2012b) have proposed several refinements, for application to time-distance (hereafter TD) methods, for the detection of subsurface signatures of emerging active regions. These include: i) dividing the annulus into a greater number of opposing arc pairs (i.e. 6, 8, 10, 12, and 14 arcs), ii) making multiple measurements with different angular orientations of each set of arcs, and iii) combining all of the TD cross-covariances made with the different arcs and their orientations before the determination of the travel times. There are four different orientations used for each arc configuration in this scheme, as each set of arcs is rotated one-quarter of the angular extent of an arc.

We explore similar procedures for $\mathrm{HH}$ using the simulation of Hartlep et al. (2011). The results here complement the tests made for $\mathrm{HH}$ on Doppler observations obtained with the MDI instrument by Braun (2012). Figure 12] shows some of our results of the measurements on the simulated perturbation. In general, the use of six arcs produces a weaker (by about 25\%) travel-time signature in the perturbation than using quadrants. A slight trend of a decreasing signal strength with the number of arcs from 6 to 14 is also observed (Figure 12 ) although the net decrease is within the background noise. The realization noise increases with the number of arcs used from about 1.4 seconds for the four orientations of the six-arc set to 2.5 seconds for the four orientations of the 14-arc set. These can be compared with the 2 second noise measured using the nominal quadrant method. These results are consistent with the increase in noise using smaller arcs observed by Braun (2012) using MDI data. The map made from combining all cross-covariances from all pupil arc configurations and orientations has a realization noise of 1.9 seconds, which is essentially identical to the nominal (quadrant) map. It is significant that maps made with different arc lengths are highly correlated with each other (Figure 12:). 
a

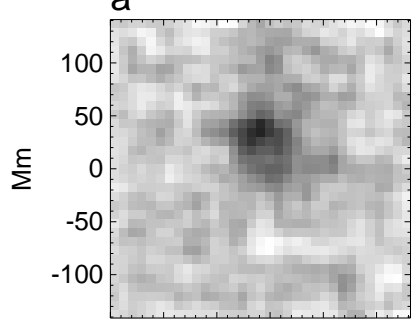

b
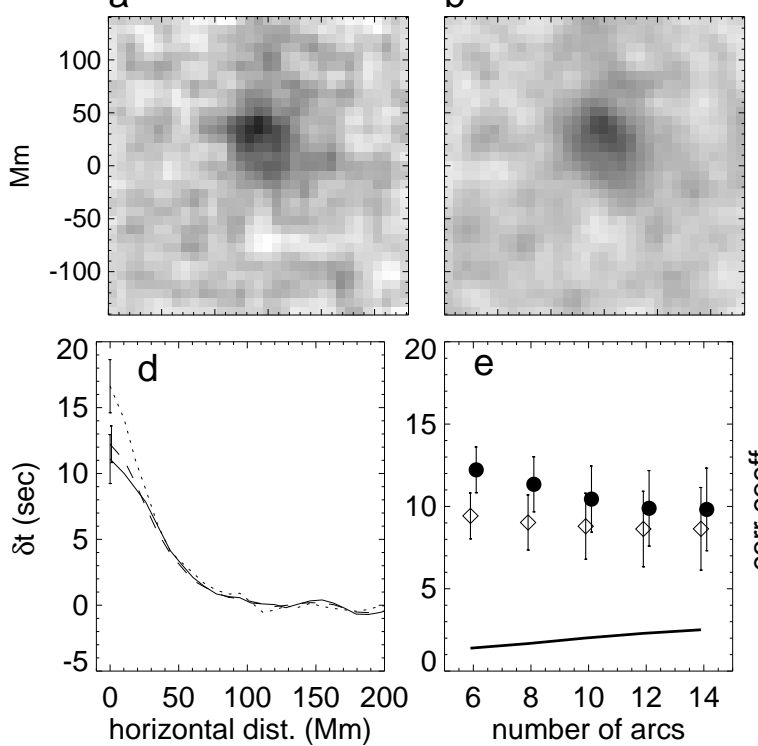
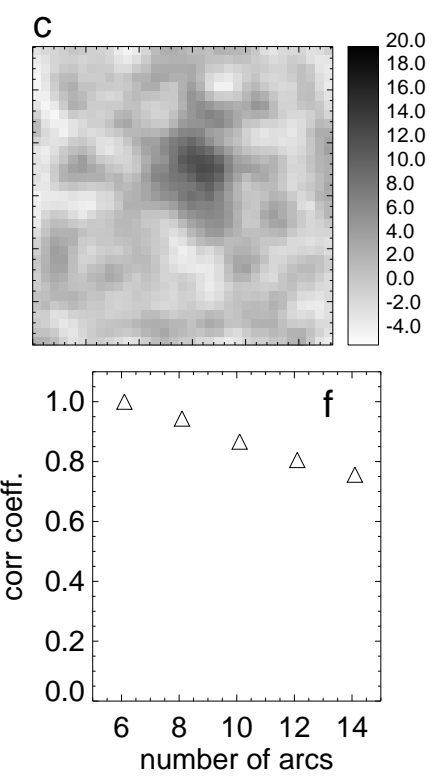

Figure 12. Mean travel time shifts at a focus depth of $54.4 \mathrm{Mm}$ from helioseismic holography using different pupil geometries: (a) the nominal method using a fixed set of four quadrant pupils, (b) using six arcs and four orientations, and (c) the combination of $6,8,10,12$, and 14 arc configurations with four orientations of each configuration. The flat-top filter shown in Figure 9 is used. (d) Azimuthal averages of the travel-time shift over annuli centered on the perturbation for the three maps shown in the three top panels: quadrants (dotted line), six arcs (dashed line), and combined 6-14 arcs (solid line). (e) Measurements of the mean travel-time shift in the perturbation and the background realization noise $\sigma$ against the number of pupil arcs used. The filled circles show the peak shift and the diamonds show the average shift within a $25 \mathrm{Mm}$ radius. Error bars denote the standard deviation of the realization noise, which is also plotted as a solid line. (f) The correlation coefficient between travel-time shift maps made using six arcs and the other arc configurations.

\section{Conclusions}

In summary, we find that helioseismic holography, as performed in the nominal lateral-vantage configuration and using the plane-parallel approximation, is in conformance with expectations well suited for detecting and characterizing subsurface sound-speed perturbations of the kind included in the simulation of Hartlep et al. (2011) at depths of at least $50 \mathrm{Mm}$. Suitable caution should be exercised since it is recognized that these results follow from a single simulation, which may have different physics from real solar perturbations. Other limitations, such as the inclusion in the simulation of only a subset (in both temporal frequencies and wavenumbers) of known solar oscillations are noted. We believe that in general, however, the viability of $\mathrm{HH}$ for the detection of subsurface perturbations is substantially confirmed, particularly its ability to select for analysis the relevant set of modes passing through a localized target below the solar surface. 
Furthermore, mindful of the caveats mentioned above, we find no evidence that the sensitivity of the procedure, as assessed by the mean travel-time shift at the expected position of the perturbation, is enhanced by the use of the flat-top filter or different pupils as suggested in the critique by Ilonidis, Zhao, and Kosovichev|l (2012a) of the results of Braun (2012). Specifically, the holographic signatures are influenced little by the detailed profile of the phase-speed filter, and, indeed, very little more by the lack of any such filter. In addition, we find that holography remains maximally sensitive when applied with spatially extended pupils, as opposed to restricting or partitioning it. The main effect of partitioning the pupil to smaller arcs is, if anything, a reduction of the signature and the appearance of diffraction effects.

Gabor-wavelet fitting can be applied to helioseismic holography as it is with other time-distance techniques, and so this should not be regarded as a discriminating qualification against it. In the case of the simulation, the results are seen to be essentially identical to the phase-method and in conformance with expected travel-time shifts given the size and amplitude of the perturbation.

Our tests do not attempt to replicate the time-distance procedures applied by Ilonidis, Zhao, and Kosovichev (2011). Thus we draw no conclusion about the sensitivity of their own measurements to the changes to methodology that they advocate. In attempting to understand the discrepancies of the results between Ilonidis, Zhao, and Kosovichev (2011) and Braun (2012), we can reasonably infer that negative holography results suggest the suspected perturbation is different than the simple sound-speed perturbation simulated by Hartlep et al. (2011). Furthermore, it would appear that the use of the plane-parallel approximation can be ruled out as a contributing factor to the negative results of Braun (2012).

It is possible that the physics of the suspected signatures may be such that, unlike a simple sound-speed perturbation, the use of narrow pupils or different filters may be critical. It is also suggested that the signatures may produce complicated changes to the cross-covariance functions, perhaps due to unknown effects of magnetic fields (Ilonidis, Zhao, and Kosovichev, 2012a). Further tests need to be performed on the relevant data. In our opinion, the possibility that the signatures of Ilonidis, Zhao, and Kosovichev (2011) represent noise also needs to be considered. We return to the point made by Braun (2012) suggesting the need for blind "hare-and-hound" tests as a minimal condition for the signatures for the signals found by Ilonidis, Zhao, and Kosovichev (2011) to be established as pre-emergence signatures of deeply submerged magnetic fields. Tests with simulated data on artificial perturbations such as reported here provide the critical context under which similar analysis of solar observations may be understood. In general the results presented here provide confidence in helioseismic holography as a useful method for probing submerged perturbations (Leka et al., 2012, Birch et al., 2012).

Acknowledgements This work is supported by NASA Heliophysics program through contract NNH12CF23C, by the NASA SDO Science Center project through contract NNH09CE41C and by the Solar Terrestrial program of the National Science Foundation through grant AGS1127327. We thank Charlie Lindsey and an anonymous referee for useful comments. We are grateful to Thomas Hartlep for providing the data used in this study. 


\section{References}

Benson, D., Stein, R., Nordlund, Å.: 2006, Supergranulation Scale Convection Simulations. In: Leibacher, J., Stein, R.F., Uitenbroek, H. (eds.) Solar MHD Theory and Observations: A High Spatial Resolution Perspective 354, Astron. Soc. Pacific, San Francisco, 92-96.

Birch, A.C., Braun, D.C., Fan, Y.: 2010, An Estimate of the Detectability of Rising Flux Tubes. Astrophys. J. 723, L190-L194.

Birch, A.C., Parchevsky, K., Braun, D.C., Kosovichev, A.: 2011, Hare and Hounds Tests of Helioseismic Holography. Solar Phys. 272, $11-28$.

Birch, A.C., Braun, D.C., Leka, K.D., Barnes, G., Javornik, B.: 2012, Helioseismology of PreEmerging Active Regions II: Average Emergence Properties. Astrophys. J., submitted.

Braun, D.C.: 1995, Sunspot Seismology: New Observations and Prospects. In: Ulrich, R.K., Rhodes, E.J. Jr., Dappen, W. (eds.) GONG 1994. Helio- and Astro-Seismology from the Earth and Space 76, Astron. Soc. Pacific, San Francisco, 250.

Braun, D.C.: 2012, Comment on "Detection of Emerging Sunspot Regions in the Solar Interior". Science 336, 296. doi 10.1126/science.1215425

Braun, D.C., Birch, A.C.: 2006, Observed Frequency Variations of Solar p-Mode Travel Times as Evidence for Surface Effects in Sunspot Seismology. Astrophys. J. 647, L187-L190.

Braun, D.C., Birch, A.C.: 2008a, Prospects for the Detection of the Deep Solar Meridional Circulation. Astrophys. J. Lett. 689, L161-L165.

Braun, D.C., Birch, A.C.: 2008b, Surface-focused Seismic Holography of Sunspots: I. Observations. Solar Phys. 251, $267-289$.

Braun, D.C., Lindsey, C.: 2000, Phase-sensitive Holography of Solar Activity. Solar Phys. 192, $307-319$.

Braun, D.C., Birch, A.C., Lindsey, C.: 2004, Local Helioseismology of Near-Surface Flows. In: Danesy, D. (ed.) SOHO 14 Helio- and Asteroseismology: Towards a Golden Future SP-559, ESA, Noordwijk, 337-340.

Braun, D.C., Birch, A.C., Benson, D., Stein, R.F., Nordlund, A.: 2007, Helioseismic Holography of Simulated Solar Convection and Prospects for the Detection of Small-Scale Subsurface Flows. Astrophys. J. 669, 1395-1405.

Braun, D.C., Birch, A.C., Rempel, M., Duvall, T.L. Jr.: 2012, Helioseismology of a Realistic Magnetoconvective Sunspot Simulation. Astrophys. J. 744, 77-86.

Cameron, R., Gizon, L., Duvall, T.L. Jr.: 2008, Helioseismology of Sunspots: Confronting Observations with Three-Dimensional MHD Simulations of Wave Propagation. Solar Phys. 251, $291-308$.

Cameron, R.H., Gizon, L., Schunker, H., Pietarila, A.: 2011, Constructing Semi-Empirical Sunspot Models for Helioseismology. Solar Phys. 268, 293-308.

Chang, H.-K., Chou, D.-Y., Sun, M.-T.: 1999, In Search of Emerging Magnetic Flux underneath the Solar Surface with Acoustic Imaging. Astrophys. J. Lett. 526, L53-L56. doi $10.1086 / 312366$

Chang, H.-K., Chou, D.-Y., Labonte, B., TON Team: 1997, Ambient acoustic imaging in helioseismology. Nature 389, 825-827. doi 10.1038/39822

Christensen-Dalsgaard, J., Dappen, W., Ajukov, S.V., Anderson, E.R., Antia, H.M., Basu, S., Baturin, V.A., Berthomieu, G., Chaboyer, B., Chitre, S.M., Cox, A.N., Demarque, P., Donatowicz, J., Dziembowski, W.A., Gabriel, M., Gough, D.O., Guenther, D.B., Guzik, J.A., Harvey, J.W., Hill, F., Houdek, G., Iglesias, C.A., Kosovichev, A.G., Leibacher, J.W., Morel, P., Proffitt, C.R., Provost, J., Reiter, J., Rhodes, E.J. Jr., Rogers, F.J., Roxburgh, I.W., Thompson, M.J., Ulrich, R.K.: 1996, The Current State of Solar Modeling. Science 272, $1286-1292$.

Couvidat, S., Birch, A.C.: 2006, Optimal Gaussian Phase-Speed Filters in Time-Distance Helioseismology. Solar Phys. 237, 229-243. doi 10.1007/s11207-006-0209-z

Crouch, A.D., Birch, A.C., Braun, D.C., Clack, C.T.M.: 2010, Helioseismic Probing of the Subsurface Structure of Sunspots. In: The Physics of Sun and Star Spots, IAU Symposium 273, 384 - 388. doi:10.1017/S1743921311015602.

Duvall, T.L. Jr., Kosovichev, A.G., Scherrer, P.H., Bogart, R.S., Bush, R.I., de Forest, C., Hoeksema, J.T., Schou, J., Saba, J.L.R., Tarbell, T.D., Title, A.M., Wolfson, C.J., Milford, P.N.: 1997, Time-Distance Helioseismology with the MDI Instrument: Initial Results. Solar Phys. 170, 63-73.

Gizon, L., Birch, A.C.: 2005, Local Helioseismology. Liv Rev Solar Phys 2. http://www.livingreviews.org/Irsp-2005-6 
Hanasoge, S.M., Larsen, R.M., Duvall, T.L. Jr., De Rosa, M.L., Hurlburt, N.E., Schou, J., Roth, M., Christensen-Dalsgaard, J., Lele, S.K.: 2006, Computational Acoustics in Spherical Geometry: Steps toward Validating Helioseismology. Astrophys. J. 648, 1268-1275.

Hartlep, T., Kosovichev, A.G., Zhao, J., Mansour, N.N.: 2011, Signatures of Emerging Subsurface Structures in Acoustic Power Maps of the Sun. Solar Phys. 268, 321 - 327.

Ilonidis, S., Zhao, J., Kosovichev, A.: 2011, Detection of Emerging Sunspot Regions in the Solar Interior. Science 333, 993 - 996. doi 10.1126/science.1206253

Ilonidis, S., Zhao, J., Kosovichev, A.: 2012a, Response to "Comment on Detection of Emerging Sunspot Regions in the Solar Interior". Science 336, 296. doi $10.1126 /$ science.1215539

Ilonidis, S., Zhao, J., Kosovichev, A.G.: 2012b, Helioseismic Detection of Emerging Magnetic Flux. In: Shibahashi, H., Takata, M., Lynas-Gray, A.E. (eds.) Progress in Solar/Stellar Physics with Helio- and Asteroseismology 462, Astron. Soc. Pacific, San Francisco, 283.

Jensen, J.M., Duvall, T.L. Jr., Jacobsen, B.H., Christensen-Dalsgaard, J.: 2001, Imaging an Emerging Active Region with Helioseismic Tomography. Astrophys. J. 553, L193-L196.

Jensen, J.M., Olsen, K.B., Duvall, T.L. Jr., Jacobsen, B.H.: 2003, Test of Helioseismic TimeDistance Inversion Using 3-D Finite-difference Wavefield Modeling. In: Sawaya-Lacoste, H. (ed.) GONG+ 2002. Local and Global Helioseismology: the Present and Future SP-517, ESA, Noordwijk, 319-320.

Kosovichev, A.G.: 2009, Photospheric and Subphotospheric Dynamics of Emerging Magnetic Flux. Space Sci. Rev. 144, 175 - 195. doi 10.1007/s11214-009-9487-8

Leka, K.D., Barnes, G., Birch, A.C., Gonzalez-Hernandez, I., Dunn, T., Javornik, B., Braun, D.C.: 2012, Helioseismology of Pre-Emerging Active Regions I: Overview, Data, and Target Selection Criteria. Astrophys. J., submitted.

Lindsey, C., Braun, D.C.: 1997, Helioseismic Holography. Astrophys. J. 485, 895-903.

Lindsey, C., Braun, D.C.: 2000, Basic Principles of Solar Acoustic Holography. Solar Phys. 192, $261-284$

Lindsey, C., Braun, D.C.: 2004, Principles of Seismic Holography for Diagnostics of the Shallow Subphotosphere. Astrophys. J. Suppl. 155, 209-225.

Markwardt, C.B.: 2009, Non-linear Least-squares Fitting in IDL with MPFIT. In: Bohlender, D.A., Durand, D., Dowler, P. (eds.) Astronomical Data Analysis Software and Systems XVIII 411, Astron. Soc. Pacific, San Francisco, 251.

Parchevsky, K.V., Kosovichev, A.G.: 2007, Three-dimensional Numerical Simulations of the Acoustic Wave Field in the Upper Convection Zone of the Sun. Astrophys. J. 666, 547558. doi $10.1086 / 520108$

Parchevsky, K.V., Kosovichev, A.G.: 2009, Numerical Simulation of Excitation and Propagation of Helioseismic MHD Waves: Effects of Inclined Magnetic Field. Astrophys. J. 694, $573-581$. doi $10.1088 / 0004-637 X / 694 / 1 / 573$

Zhao, J., Georgobiani, D., Kosovichev, A.G., Benson, D., Stein, R.F., Nordlund, Å.: 2007, Validation of Time-Distance Helioseismology by Use of Realistic Simulations of Solar Convection. Astrophys. J. 659, 848-857.

Zharkov, S., Thompson, M.J.: 2008, Time Distance Analysis of the Emerging Active Region NOAA 10790. Solar Phys. 251, 369-380. doi 10.1007/s11207-008-9239-z 
SOLA: ms.tex; 28 August 2018; 12:18; p. 20 